\title{
TWO NUMERICAL APPROACHES TO STATIONARY MEAN-FIELD GAMES
}

\author{
NOHA ALMULLA, RITA FERREIRA, AND DIOGO GOMES
}

\begin{abstract}
Here, we consider numerical methods for stationary mean-field games (MFG) and investigate two classes of algorithms. The first one is a gradient-flow method based on the variational characterization of certain MFG. The second one uses monotonicity properties of MFG. We illustrate our methods with various examples, including onedimensional periodic MFG, congestion problems, and higher-dimensional models.
\end{abstract}

\section{INTRODUCTION}

Mean-field games (MFG) model problems with a large number of rational agents interacting non-cooperatively [37, 38, 39, 36, 35. Much progress has been achieved in the mathematical theory of MFG for time-dependent problems [43, 44, 12, 23, 22, 21, 20, 30, 27. and for stationary problems [24, 28, 46, 29, 19, 42] (also see the recent surveys [10, 31, 7]). Yet, in the absence of explicit solutions, the efficient simulation of MFG is importance to many applications. Consequently, researchers have studied numerical algorithms in various cases, including continuous state problems [5, 1, 2, 3, 4, 34, 32, 13, 8, 33, 9, and finite state problems [25, 26. Here, we develop numerical methods for (continuous state) stationary MFG using variational and monotonicity methods.

Certain MFG, called variational MFG, are Euler-Lagrange equations of integral functionals. These MFG are instances of a wider class of problems - monotonic MFG. In the context of numerical methods, the variational structure of MFG was explored in [2. Moreover, monotonicity properties are critical for the convergence of the methods in [1, 3, 4]. Recently, variational and monotonicity methods were used to prove the existence of weak solutions to MFG in, respectively, [40, 45, 11, 12] and [17.

Here, our main contributions are two computational approaches for MFG. For variational MFG, we build an approximating method using a gradient flow approach. This technique gives a simple and efficient algorithm. Nevertheless, the class of variational MFG is somewhat restricted. Monotonic MFG encompass a wider range of problems that include variational MFG as a particular case. In these games, the MFG equations involve a monotone nonlinear operator. We use the monotonicity to build a flow that is a contraction in $L^{2}$ and whose fixed points solve the MFG.

To keep the presentation elementary, we develop our methods for the one-dimensional MFG:

$$
\left\{\begin{array}{l}
\frac{u_{x}^{2}}{2}+V(x)+b(x) u_{x}=\ln m+\bar{H}, \\
-\left(m\left(u_{x}+b(x)\right)\right)_{x}=0 .
\end{array}\right.
$$

To streamline the discussion, we study (1.1) with periodic boundary conditions. Thus, the variable $x$ takes values in the one-dimensional torus, $\mathbb{T}$. The potential, $V$, and the drift, $b$, are given real-valued periodic functions. The unknowns are $u, m$, and $\bar{H}$, where $u$ and $m$ are real-valued periodic functions satisfying $m>0$, and where $\bar{H}$ is a constant. The role of $\bar{H}$ is to allow for $m$ to satisfy $\int_{\mathbb{T}} m \mathrm{~d} x=1$. Furthermore, since adding an arbitrary constant to $u$ does not change 1.1), we require

$$
\int_{\mathbb{T}} u \mathrm{~d} x=0 .
$$

The authors were partially supported by King Abdullah University of Science and Technology baseline and start-up funds and by KAUST SRI, Center for Uncertainty Quantification in Computational Science and Engineering. 
The system (1.1) is one of the simplest MFG models. However, its structure is quite rich and illustrates our techniques well. Our methods extend in a straightforward way to other models, including higher-dimensional problems. In particular, in Section 4 we discuss applications to a one-dimensional congestion model and to a two-dimensional MFG.

We end this introduction with a brief outline of our work. In Section 2, we examine various properties of (1.1). These properties motivate the ideas used in Section 3 to build numerical methods. Next, in Section 4, we discuss the implementation of our approaches and present their numerical simulations. We conclude this work in Section 5 with some final remarks.

\section{Elementary properties}

We begin this section by constructing explicit solutions to 1.1 . These are of particular importance for the validation and comparison of the numerical methods presented in Section 3. Next, we discuss the variational structure of (1.1) and show that 1.1 is equivalent to the Euler-Lagrange equation of a suitable functional. Because of this, we introduce a gradient flow approximation and examine some of its elementary properties. Finally, we explain how 1.1 can be seen as a monotone operator. This operator induces a flow that is a contraction in $L^{2}$ and whose stationary points are solutions to (1.1).

2.1. Explicit solutions. Here, we build explicit solutions to 1.1. For simplicity, we assume that $V$ and $b$ are $C^{\infty}$ functions. Moreover, we identify $\mathbb{T}$ with the interval $[0,1]$.

Due to the one-dimensional nature of $(1.1)$, if $\int_{\mathbb{T}} b \mathrm{~d} x=0$, we have the following explicit solution

$$
\begin{aligned}
& u(x)=-\int_{0}^{x} b(y) \mathrm{d} y+\int_{\mathbb{T}} \int_{0}^{x} b(y) \mathrm{d} y \mathrm{~d} x, \quad m(x)=\frac{e^{V(x)-\frac{b^{2}(x)}{2}}}{\int_{\mathbb{T}} e^{V(y)-\frac{b^{2}(y)}{2}} \mathrm{~d} y}, \\
& \bar{H}=\ln \left(\int_{\mathbb{T}} e^{V(y)-\frac{b^{2}(y)}{2}} \mathrm{~d} y\right) .
\end{aligned}
$$

Suppose that $b=\psi_{x}$ for some $C^{\infty}$ and periodic function $\psi: \mathbb{T} \rightarrow \mathbb{R}$ with $\int_{\mathbb{T}} \psi \mathrm{d} x=0$. For

$$
u(x)=\psi(x), \quad m(x)=\frac{e^{V(x)-\frac{\psi_{x}^{2}(x)}{2}}}{\int_{\mathbb{T}} e^{V(y)-\frac{\psi_{y}^{2}(y)}{2}} \mathrm{~d} y}, \quad H=\ln \left(\int_{\mathbb{T}} e^{V(y)-\frac{\psi_{y}^{2}(y)}{2}} \mathrm{~d} y\right),
$$

the triplet $(u, m, \bar{H})$ solves 1.1 . If $\int_{\mathbb{T}} b \mathrm{~d} x \neq 0$, we are not aware of any closed-form solution.

Next, we consider the congestion model

$$
\left\{\begin{array}{l}
\frac{u_{x}^{2}}{2 m^{1 / 2}}+V(x)=\ln m+\bar{H} \\
-\left(m^{1 / 2} u_{x}\right)_{x}=0
\end{array}\right.
$$

Remarkably, the previous equation has the same solutions as 1.1 with $b=0$. Namely, for $u(x)=0, m(x)=\frac{e^{V(x)}}{\int_{\mathbb{T}} e^{V(y)} \mathrm{d} y}$, and $\bar{H}=\ln \left(\int_{\mathbb{T}} e^{V(y)} \mathrm{d} y\right)$, the triplet $(u, m, \bar{H})$ solves 2.1.

2.2. Euler-Lagrange equation. We begin by showing that 1.1 is equivalent to the EulerLagrange equation of the integral functional

$$
J[u]=\int_{\mathbb{T}} e^{\frac{u_{x}^{2}}{2}+V(x)+b(x) u_{x}} \mathrm{~d} x
$$

defined for $u \in D(J)=W^{2,2}(\mathbb{T}) \cap L_{0}^{2}(\mathbb{T})$, where $L_{0}^{2}(\mathbb{T})=\left\{u \in L^{2}(\mathbb{T}): u\right.$ satisfies $[1.2\}$.

Remark 2.1 (On the domain of $J$ ). As proved in [15] (see [16, [18, 19, 23, 24, and [42 for related problems), 1.1 admits a $C^{\infty}$ solution. By a simple convexity argument, this solution is the unique minimizer of

$$
J[u]=\min _{v \in W^{1,2}(\mathbb{T}), \int_{\mathbb{T}} v \mathrm{~d} x=0} J[v] .
$$

Thus, the minimizers of $J$ in $\left\{v \in W^{1,2}(\mathbb{T}): \int_{\mathbb{T}} v \mathrm{~d} x=0\right\}$ are also minimizers of $J$ in $\left\{v \in W^{2,2}(\mathbb{T}): \int_{\mathbb{T}} v \mathrm{~d} x=0\right\}$. Thus, the domain of $J$ is not too restrictive, and, due to 
this choice, our arguments are substantially simplified. In particular, because we are in the one-dimensional setting, $W^{2,2}(\mathbb{T}) \subset W^{1, \infty}(\mathbb{T})$.

We also observe that $L_{0}^{2}(\mathbb{T})$ endowed with the $L^{2}(\mathbb{T})$-inner product is a Hilbert space.

Lemma 2.2. For $\bar{H}=0,(1.1)$ is equivalent to the Euler-Lagrange equation of $J$.

Proof. Let $u \in W^{2,2}(\mathbb{T}) \cap L_{0}^{2}(\mathbb{T})$. We say that $u$ is a critical point of $J$ if

$$
\frac{\mathrm{d}}{\mathrm{d} \varepsilon} J[u+\varepsilon v]_{\left.\right|_{\varepsilon=0}}=0
$$

for all $v \in W^{2,2}(\mathbb{T}) \cap L_{0}^{2}(\mathbb{T})$. Fix any such $v$. For all $\varepsilon \in \mathbb{R}$, we have that

$$
\frac{\mathrm{d}}{\mathrm{d} \varepsilon} J[u+\varepsilon v]=\int_{\mathbb{T}} e^{\frac{u_{x}^{2}}{2}+\varepsilon u_{x} v_{x}+\varepsilon^{2} \frac{v_{x}^{2}}{2}+V(x)+b(x) u_{x}+\varepsilon b(x) v_{x}}\left(u_{x} v_{x}+b(x) v_{x}+\varepsilon v_{x}^{2}\right) \mathrm{d} x .
$$

Define $m$ by

$$
\ln m=\frac{u_{x}^{2}}{2}+V(x)+b(x) u_{x}
$$

Then, it follows that

$$
\frac{\mathrm{d}}{\mathrm{d} \varepsilon} J[u+\varepsilon v]_{\varepsilon=0}=0 \Leftrightarrow \int_{\mathbb{T}} m\left(u_{x}+b(x)\right) v_{x} \mathrm{~d} x=0 \Leftrightarrow-\int_{\mathbb{T}}\left(m\left(u_{x}+b(x)\right)\right)_{x} v \mathrm{~d} x=0 .
$$

Since $v \in W^{2,2}(\mathbb{T})$ is an arbitrary function with zero mean, we conclude that $u$ is a critical point of $J$ if, and only if, $(m, u)$ satisfies 1.1 .

As mentioned in Remark 2.1 the functional $J$ defined by (2.2) admits a unique minimizer. Moreover, since $J$ is convex, any solution to the associated Euler-Lagrange equation is a minimizer. By 2.3), we have $m>0$. In MFG, it is usual to require

$$
\int_{\mathbb{T}} m \mathrm{~d} x=1 .
$$

To normalize $m$, we multiplying $m$ by a suitable constant and introduce the parameter $\bar{H}$, which leads us to 1.1 .

2.3. Monotonicity conditions. Let $H$ be a Hilbert space with the inner product $\langle\cdot, \cdot\rangle_{H}$. A map $A: D(A) \subset H \rightarrow H$ is a monotone operator if

$$
\langle A(w)-A(\tilde{w}), w-\tilde{w}\rangle_{H} \geqslant 0,
$$

for all $w, \tilde{w} \in D(A)$.

In the Hilbert space $L^{2}(\mathbb{T}) \times L^{2}(\mathbb{T})$, we define

$$
A\left[\begin{array}{c}
m \\
u
\end{array}\right]=\left[\begin{array}{c}
-\frac{u_{x}^{2}}{2}-V(x)-b(x) u_{x}+\ln m \\
-\left(m\left(u_{x}+b(x)\right)\right)_{x}
\end{array}\right],
$$

with $D(A)=\left\{(m, u) \in W^{1,2}(\mathbb{T}) \times W^{2,2}(\mathbb{T}): \inf _{\mathbb{T}} m>0\right\}$. Observe that $A$ maps $D(A)$ into $L^{2}(\mathbb{T}) \times L^{2}(\mathbb{T})$ because $W^{1,2}(\mathbb{T})$ is continuously embedded in $L^{\infty}(\mathbb{T})$.

Lemma 2.3. The operator $A$ given by 2.4 is a monotone operator in $L^{2}(\mathbb{T}) \times L^{2}(\mathbb{T})$.

Proof. Let $(m, u),(\theta, v) \in D(A) \subset L^{2}(\mathbb{T}) \times L^{2}(\mathbb{T})$. We have

$$
\begin{aligned}
\left\langle A\left[\begin{array}{l}
m \\
u
\end{array}\right]-A\left[\begin{array}{l}
\theta \\
v
\end{array}\right],\left[\begin{array}{l}
m \\
u
\end{array}\right]-\left[\begin{array}{l}
\theta \\
v
\end{array}\right]\right\rangle_{L^{2}(\mathbb{T}) \times L^{2}(\mathbb{T})} \\
=\int_{\mathbb{T}}(\ln m-\ln \theta)(m-\theta) \mathrm{d} x+\int_{\mathbb{T}}\left(\frac{m}{2}+\frac{\theta}{2}\right)\left(u_{x}-v_{x}\right)^{2} \mathrm{~d} x,
\end{aligned}
$$

where we used integration by parts. Because $\ln (\cdot)$ is an increasing function, and because $\theta, m>0$, the conclusion follows.

As observed in [37, 39], the monotonicity of $A$ implies the uniqueness of the solutions. Here, we use the monotonicity to construct a flow that approximates solutions of (1.1). 
2.4. Weak solutions. Denote by $\langle\cdot, \cdot\rangle_{\mathcal{D} \times \mathcal{D}^{\prime}}$ the duality pairing in the sense of distributions. We say that a triplet $(m, u, \bar{H}) \in \mathcal{D}^{\prime} \times \mathcal{D}^{\prime} \times \mathbb{R}$ is a weak solution of (1.1) if

$$
\left\langle A\left[\begin{array}{l}
\theta \\
v
\end{array}\right]-\left[\begin{array}{c}
\bar{H} \\
0
\end{array}\right],\left[\begin{array}{l}
\theta \\
v
\end{array}\right]-\left[\begin{array}{c}
m \\
u
\end{array}\right]\right\rangle_{\mathcal{D} \times \mathcal{D}^{\prime}} \geqslant 0
$$

for all $(\theta, v) \in \mathcal{D} \times \mathcal{D}$ satisfying $\inf _{\mathbb{T}} \theta>0$ and $\int_{\mathbb{T}} \theta \mathrm{d} x=1$.

2.5. Continuous gradient flow. Next, we introduce the gradient flow of the energy 2.2 with respect to the $L^{2}(\mathbb{T})$-inner product. First, we extend $J$ in $(2.2)$ to the whole space $L_{0}^{2}(\mathbb{T})$ by setting $J[u]=+\infty$ if $u \in L_{0}^{2}(\mathbb{T}) \backslash W^{2,2}(\mathbb{T})$. We will not relabel this extension.

The functional $J: L_{0}^{2}(\mathbb{T}) \rightarrow[0,+\infty]$ is proper, convex, and lower semicontinuous in $L_{0}^{2}(\mathbb{T})$. The subdifferential of $J$ is the map $\partial J: L_{0}^{2}(\mathbb{T}) \rightarrow 2^{L_{0}^{2}(\mathbb{T})}$ defined for $u \in L_{0}^{2}(\mathbb{T})$ by

$$
\partial J[u]=\left\{v \in L_{0}^{2}(\mathbb{T}): J[w] \geqslant J[u]+\langle v, w-u\rangle_{L^{2}(\mathbb{T})} \text { for all } w \in L_{0}^{2}(\mathbb{T})\right\} .
$$

The domain of $\partial J, D(\partial J)$, is the set of all $u \in L_{0}^{2}(\mathbb{T})$ such that $\partial J[u] \neq \emptyset$.

The gradient flow with respect to the $L^{2}(\mathbb{T})$-inner product and energy $J$ is

$$
\dot{\boldsymbol{u}}(t) \in-\partial J[\boldsymbol{u}(t)], \quad t \geqslant 0,
$$

where $\boldsymbol{u}:[0,+\infty) \rightarrow L_{0}^{2}(\mathbb{T})$. As we will see next, 2.5 is equivalent to

$$
\dot{\boldsymbol{u}}(t)=\left(\boldsymbol{m}(t)\left((\boldsymbol{u}(t))_{x}+b(x)\right)\right)_{x}, \quad t \geqslant 0,
$$

where $\boldsymbol{m}(t)$ is given by (2.3) with $u$ replaced by $\boldsymbol{u}(t)$. Moreover, if the solution $\boldsymbol{u}$ to 2.6 is regular enough, then

$$
\frac{\mathrm{d}}{\mathrm{d} t} J[\boldsymbol{u}]=-\int_{\mathbb{T}}\left[\left(\boldsymbol{m}\left(\boldsymbol{u}_{x}+b(x)\right)\right)_{x}\right]^{2} \mathrm{~d} x \leqslant 0 .
$$

Proposition 2.4. We have $D(\partial J)=W^{2,2}(\mathbb{T}) \cap L_{0}^{2}(\mathbb{T})$ and, for $u \in D(\partial J)$, $\partial J[u]=$ $-\left(m\left(u_{x}+b(x)\right)\right)_{x}$, where $m$ is given by 2.3 .

Proof. By [14, Thm. 1 in $\S 9.6 .1], D(\partial J) \subset D(J)=\left\{u \in L_{0}^{2}(\mathbb{T}): J[u]<+\infty\right\}=W^{2,2}(\mathbb{T}) \cap$ $L_{0}^{2}(\mathbb{T})$.

Conversely, fix $u \in W^{2,2}(\mathbb{T}) \cap L_{0}^{2}(\mathbb{T})$, let $m$ be given by $(2.3)$, and set $v=-\left(m\left(u_{x}+b(x)\right)\right)_{x}$. Then, $v \in L_{0}^{2}(\mathbb{T})$ by the embedding $W^{2,2}(\mathbb{T}) \subset W^{1, \infty}(\mathbb{T})$ and by the periodicity of $u, m$, and $b$. Moreover, using the convexity of the exponential function, the integration by parts formula, and the conditions $m>0$ and $\frac{w_{x}^{2}}{2}-\frac{u_{x}^{2}}{2} \geqslant u_{x} w_{x}-u_{x}^{2}$, for each $w \in W^{2,2}(\mathbb{T}) \cap L_{0}^{2}(\mathbb{T})$, we obtain

$$
\begin{aligned}
J[w] & \geqslant J[u]+\int_{\mathbb{T}} m\left(\frac{w_{x}^{2}}{2}+b(x) w_{x}-\frac{u_{x}^{2}}{2}-b(x) u_{x}\right) \mathrm{d} x \\
& \geqslant J[u]+\int_{\mathbb{T}} m\left(u_{x} w_{x}-u_{x}^{2}+b(x)\left(w_{x}-u_{x}\right)\right) \mathrm{d} x \\
& =J[u]-\int_{\mathbb{T}}\left(m\left(u_{x}+b(x)\right)\right)_{x}(w-u) \mathrm{d} x .
\end{aligned}
$$

Because $w \in W^{2,2}(\mathbb{T}) \cap L_{0}^{2}(\mathbb{T})$ is arbitrary, and because $J=+\infty$ in $L_{0}^{2}(\mathbb{T}) \backslash W^{2,2}(\mathbb{T})$, we obtain $v=-\left(m\left(u_{x}+b(x)\right)\right)_{x} \in \partial J[u]$. Because $u \in W^{2,2}(\mathbb{T}) \cap L_{0}^{2}(\mathbb{T})$ is also arbitrary, we get $D(\partial J) \supset W^{2,2}(\mathbb{T}) \cap L_{0}^{2}(\mathbb{T})$.

To conclude the proof, we show that for $u \in D(\partial J)$, the function $-\left(m\left(u_{x}+b(x)\right)\right)_{x}$ with $m$ given by $(2.3)$ is the unique element of $\partial J[u]$. Let $\bar{v} \in \partial J[u]$. Then, for all $\varepsilon>0$ and $w \in W^{2,2}(\mathbb{T}) \cap L_{0}^{2}(\mathbb{T})$, we have

$$
\frac{J[u \pm \varepsilon w]-J[u]}{\varepsilon} \geqslant \pm\langle\bar{v}, w\rangle_{L^{2}(\mathbb{T})} .
$$

Letting $\varepsilon \rightarrow 0^{+}$and arguing as in the proof of Lemma 2.2, we obtain

$$
\left\langle-\left(m\left(u_{x}+b(x)\right)\right)_{x}, w\right\rangle_{L^{2}(\mathbb{T})} \geqslant \pm\langle\bar{v}, w\rangle_{L^{2}(\mathbb{T})} .
$$

Because $w \in W^{2,2}(\mathbb{T}) \cap L_{0}^{2}(\mathbb{T})$ is arbitrary, it follows that $\bar{v}=-\left(m\left(u_{x}+b(x)\right)\right)_{x}$. 
The following result about solutions to the gradient flow $(2.6)$ holds by [14, Thm. 3 in $\S 9.6 .2]$ and by the fact that $W^{2,2}(\mathbb{T}) \cap L_{0}^{2}(\mathbb{T})$ is dense in $L_{0}^{2}(\mathbb{T})$.

Theorem 2.5. For each $u \in W^{2,2}(\mathbb{T}) \cap L_{0}^{2}(\mathbb{T})$, there exists a unique function $\boldsymbol{u} \in C\left([0,+\infty) ; L_{0}^{2}(\mathbb{T})\right)$, with $\dot{\boldsymbol{u}} \in L^{\infty}\left(0,+\infty ; L_{0}^{2}(\mathbb{T})\right)$, such that

(i) $\boldsymbol{u}(0)=u$,

(ii) $\boldsymbol{u}(t) \in W^{2,2}(\mathbb{T}) \cap L_{0}^{2}(\mathbb{T})$ for each $t>0$,

(iii) $\dot{\boldsymbol{u}}(t)=\left(\boldsymbol{m}(t)\left((\boldsymbol{u}(t))_{x}+b(x)\right)\right)_{x}$ for a.e. $t \geqslant 0$, where $\boldsymbol{m}(t)$ is given by (2.3) with $u$ replaced by $\boldsymbol{u}(t)$.

2.6. Monotonic flow. Because the operator $A$ is monotone, the flow

$$
\left[\begin{array}{c}
\dot{\boldsymbol{m}} \\
\dot{\boldsymbol{u}}
\end{array}\right]=-A\left[\begin{array}{c}
\boldsymbol{m} \\
\boldsymbol{u}
\end{array}\right]
$$

is a contraction in $L^{2}(\mathbb{T}) \times L^{2}(\mathbb{T})$. That is, if $(\boldsymbol{m}, \boldsymbol{u})$ and $(\tilde{\boldsymbol{m}}, \tilde{\boldsymbol{u}})$ solve $(2.7)$, then

$$
\frac{\mathrm{d}}{\mathrm{d} t}\left(\|\boldsymbol{u}-\tilde{\boldsymbol{u}}\|_{L^{2}(\mathbb{T})}^{2}+\|\boldsymbol{m}-\tilde{\boldsymbol{m}}\|_{L^{2}(\mathbb{T})}^{2}\right)=-2\left\langle A\left[\begin{array}{c}
\boldsymbol{m} \\
\boldsymbol{u}
\end{array}\right]-A\left[\begin{array}{c}
\tilde{\boldsymbol{m}} \\
\tilde{\boldsymbol{u}}
\end{array}\right],\left[\begin{array}{c}
\boldsymbol{m} \\
\boldsymbol{u}
\end{array}\right]-\left[\begin{array}{c}
\tilde{\boldsymbol{m}} \\
\tilde{\boldsymbol{u}}
\end{array}\right]\right\rangle_{L^{2}(\mathbb{T}) \times L^{2}(\mathbb{T})} \leqslant 0,
$$

provided that for each $t \geqslant 0,(\boldsymbol{m}(t), \boldsymbol{u}(t)),(\tilde{\boldsymbol{m}}(t), \tilde{\boldsymbol{u}}(t)) \in D(A)$. The flow (2.7) has two undesirable features. First, it does not preserve probabilities; second, the flow may not preserve the condition $\boldsymbol{m}>0$. To conserve probability, we modify 2.7 through

$$
\left[\begin{array}{c}
\dot{\boldsymbol{m}} \\
\dot{\boldsymbol{u}}
\end{array}\right]=-A\left[\begin{array}{c}
\boldsymbol{m} \\
\boldsymbol{u}
\end{array}\right]+\left[\begin{array}{c}
\bar{H}(t) \\
0
\end{array}\right]
$$

where $\bar{H}(t)$ is such that $\frac{\mathrm{d}}{\mathrm{d} t} \int_{\mathbb{T}} \boldsymbol{m} \mathrm{d} x=0$. A straightforward computation shows that (2.8) is still a contraction in $L^{2}(\mathbb{T}) \times L^{2}(\mathbb{T})$. More precisely, if $(\boldsymbol{m}, \boldsymbol{u})$ and $(\tilde{\boldsymbol{m}}, \tilde{\boldsymbol{u}})$ solve (2.8) and satisfy $(\boldsymbol{m}(t), \boldsymbol{u}(t)),(\tilde{\boldsymbol{m}}(t), \tilde{\boldsymbol{u}}(t)) \in D(A)$ for all $t \geqslant 0, \int_{\mathbb{T}} \boldsymbol{m}(0) \mathrm{d} x=1$, and $\int_{\mathbb{T}} \tilde{\boldsymbol{m}}(0) \mathrm{d} x=1$, then

$$
\frac{\mathrm{d}}{\mathrm{d} t}\left(\|\boldsymbol{u}-\tilde{\boldsymbol{u}}\|_{L^{2}(\mathbb{T})}^{2}+\|\boldsymbol{m}-\tilde{\boldsymbol{m}}\|_{L^{2}(\mathbb{T})}^{2}\right) \leqslant 0 .
$$

Furthermore, positivity holds for the discretization of 2.8 that we develop in the next section. Therefore, the discrete analog of $(2.8)$ is a contracting flow that preserves probability and positivity. Then, as $t \rightarrow \infty$, the solutions approximate 1.1 .

\section{DiscRete SETTING}

Here, we discuss the numerical approximation of 1.1 . We use a monotone scheme for the Hamilton-Jacobi equation. For the Fokker-Planck equation, we consider the adjoint of the linearization of the discrete Hamilton-Jacobi equation. This technique preserves both the gradient structure and the monotonicity properties of the original problem.

3.1. Discretization of the Hamilton-Jacobi operator. We consider $N$ equidistributed points on $[0,1], x_{i}=\frac{i}{N}, i \in\{1, \ldots, N\}$, and corresponding values of the approximation to $u$ given by the vector $u=\left(u_{1}, \ldots, u_{N}\right) \in \mathbb{R}^{N}$. We set $h=\frac{1}{N}$. To incorporate the periodic conditions, we use the periodicity convention $u_{0}=u_{N}$ and $u_{N+1}=u_{1}$. For each $i \in\{1, \ldots, N\}$, let $\psi_{i}: \mathbb{R}^{N} \rightarrow \mathbb{R}^{2}$ be given by

$$
\psi_{i}(u)=\left(\psi_{i}^{1}(u), \psi_{i}^{2}(u)\right)=\left(\frac{u_{i}-u_{i+1}}{h}, \frac{u_{i}-u_{i-1}}{h}\right)
$$

for $u \in \mathbb{R}^{N}$. To discretize the operator

$$
u \mapsto \frac{u_{x}^{2}}{2}+V(x)+b(x) u_{x}
$$

we use a monotone finite difference scheme, see [6]. This scheme is built as follows. We consider a function $F: \mathbb{R} \times \mathbb{R} \times \mathbb{T} \rightarrow \mathbb{R}$ satisfying the following four conditions.

1. $F(p, q, x)$ is jointly convex in $(p, q)$.

2. The functions $p \mapsto F(p, q, x)$ for fixed $(q, x)$ and $q \mapsto F(p, q, x)$ for fixed $(p, x)$ are increasing.

3. $F(-p, p, x)=\frac{p^{2}}{2}+b(x) p+V(x)$. 
4. There exists a positive constant, $c$, such that

$$
F(-p, q, x)+F\left(q^{\prime}, p, x^{\prime}\right) \geqslant-\frac{1}{c}+c p^{2} .
$$

An example of such a function may be found in Section 4 below. Next, we set

$$
F_{i}(p, q)=F\left(p, q, x_{i}\right) .
$$

Let $G: \mathbb{R}^{N} \rightarrow \mathbb{R}^{N}$ be the function defined for $u \in \mathbb{R}^{N}$ by

$$
G(u)=\left(G_{1}(u), \ldots, G_{N}(u)\right)=\left(\left(F_{1} \circ \psi_{1}\right)(u), \ldots,\left(F_{N} \circ \psi_{N}\right)(u)\right) .
$$

Then, $G(u)$ is a finite difference scheme for the Hamilton-Jacobi operator $\frac{u_{x}^{2}}{2}+V(x)+b(x) u_{x}$.

Remark 3.1. In the higher-dimensional case, the Hamilton-Jacobi operator can be discretized with a similar monotone scheme. See [41] for a systematic study of convergent monotone difference schemes for elliptic and parabolic equations.

3.2. The variational formulation. Here, we study the following discrete version, $\phi$ : $\mathbb{R}^{N} \rightarrow \mathbb{R}$, of the functional 2.2 :

$$
\phi(u)=\sum_{i=1}^{N} h e^{G_{i}(u)}, \quad u \in \mathbb{R}^{N},
$$

where $G_{i}$ is given by 3.3 .

Lemma 3.2. The function $\phi$ given by (3.4) is convex.

Proof. Fix $\lambda \in(0,1)$ and $u, v \in \mathbb{R}^{N}$. Because each $F_{i}$ is convex, because the exponential is an increasing convex function, and because $h>0$, we have

$$
\begin{aligned}
\phi(\lambda u+(1-\lambda) v) & =\sum_{i=1}^{N} h e^{F_{i}\left(\lambda \frac{u_{i}-u_{i+1}}{h}+(1-\lambda) \frac{v_{i}-v_{i+1}}{h}, \lambda \frac{u_{i}-u_{i-1}}{h}+(1-\lambda) \frac{v_{i}-v_{i-1}}{h}\right)} \\
& \leqslant \sum_{i=1}^{N} h e^{\lambda F_{i}\left(\frac{u_{i}-u_{i+1}}{h}, \frac{u_{i}-u_{i-1}}{h}\right)+(1-\lambda) F_{i}\left(\frac{v_{i}-v_{i+1}}{h}, \frac{v_{i}-v_{i-1}}{h}\right)} \\
& \leqslant \lambda \phi(u)+(1-\lambda) \phi(v),
\end{aligned}
$$

which completes the proof.

Lemma 3.3. Let $\phi$ be given by (3.4). Let $\mathcal{L}_{u}^{*}: \mathbb{R}^{N} \rightarrow \mathbb{R}^{N}$ represent the adjoint operator of the linearized operator $\mathcal{L}_{u}: \mathbb{R}^{N} \rightarrow \mathbb{R}^{N}$ of the function $G$ at $u \in \mathbb{R}^{N}$. A vector $u \in \mathbb{R}^{N}$ is a critical point of $\phi$ if and only if there exists $\tilde{m} \in \mathbb{R}_{+}^{N}$ such that the pair $(\tilde{m}, u)$ satisfies

$$
\left\{\begin{array}{l}
G_{i}(u)=\ln \tilde{m}_{i} \\
\left(\mathcal{L}_{u}^{*} \tilde{m}\right)_{i}=0
\end{array}\right.
$$

for all $i \in\{1, \ldots, N\}$.

Proof. The proof is similar to the one for Lemma 2.2 .

Remark 3.4. We observe that for $w \in \mathbb{R}^{N}$ and $i \in\{1, \ldots, N\}$, we have

$$
\begin{aligned}
\left(\mathcal{L}_{u}^{*} w\right)_{i} & =\sum_{j=1}^{N} \partial_{i} G_{j}(u) w_{j}=\sum_{j=i-1}^{i+1} \frac{\partial}{\partial u_{i}} F_{j}\left(\psi_{j}(u)\right) w_{j} \\
& =\frac{1}{h}\left[-\frac{\partial F_{i-1}}{\partial p}\left(\psi_{i-1}(u)\right) w_{i-1}+\frac{\partial F_{i}}{\partial p}\left(\psi_{i}(u)\right) w_{i}+\frac{\partial F_{i}}{\partial q}\left(\psi_{i}(u)\right) w_{i}-\frac{\partial F_{i+1}}{\partial q}\left(\psi_{i+1}(u)\right) w_{i+1}\right] .
\end{aligned}
$$

Simple computations show that $\mathcal{L}_{u}^{*} w$ is a consistent finite difference scheme for the FokkerPlanck equation. 
3.3. The discretized operator. Motivated by the previous discussion, we discretize 1.1 through the finite difference operator

$$
A^{N}\left[\begin{array}{c}
m \\
u
\end{array}\right]=\left[\begin{array}{c}
-G(u)+\ln m \\
\mathcal{L}_{u}^{*} m
\end{array}\right], \quad(m, u) \in \mathbb{R}_{+}^{N} \times \mathbb{R}^{N},
$$

where $\ln m=\left(\ln m_{1}, \ldots, \ln m_{N}\right)$ and where $G$ is given by (3.3). Accordingly, the analog to (1.1) becomes

$$
A^{N}\left[\begin{array}{c}
m^{N} \\
u^{N}
\end{array}\right]=\left[\begin{array}{c}
-\bar{H}^{N} \iota \\
0
\end{array}\right]
$$

where we highlighted the dependence on $N$ and where $\iota=(1, \ldots, 1) \in \mathbb{R}^{N}$. In $(\sqrt{3.6})$, the unknowns are the vector $u^{N}$, the discrete probability density $m^{N}$, normalized to $h \sum_{i=1}^{N} m_{i}^{N}=$ 1, and the effective Hamiltonian $\bar{H}^{N}$.

We are interested in two main points. The first is the existence and approximation of solutions to (3.6). The second is the convergence of these solutions to solutions of (1.1). The first issue will be examined by gradient-flow techniques and by monotonicity methods. The second issue is a consequence of a modified Minty method.

3.4. Existence of solutions. Here, we prove the existence of solutions to (3.6). Our proof uses ideas similar to those of the direct method of the calculus of variations.

Proposition 3.5. Let $\phi$ be as in (3.4). Then, there exists $u^{N} \in \mathbb{R}^{N}$ with $\sum_{i=1}^{N} u_{i}^{N}=0$ that minimizes $\phi$. Moreover,

$$
h \sum_{i=1}^{N}\left(u_{i}^{N}\right)^{2} \leqslant C
$$

for some positive constant $C$ independent of $h$. In addition, there exist $m^{N} \in \mathbb{R}^{N}$ with $h \sum_{i=1}^{N} m_{i}^{N}=1$ and $\bar{H}^{N} \in \mathbb{R}^{N}$ such that the triplet $\left(u^{N}, m^{N}, \bar{H}^{N}\right)$ satisfies 3.6 .

Proof. To simplify the notation, we will drop the explicit dependence on $N$ of $u^{N}$ and $m^{N}$. Accordingly, we simply write $u$ and $m$.

As in the direct method of the calculus of variations, we select a minimizing sequence, $\left(u^{k}\right)_{k \in \mathbb{N}} \subset \mathbb{R}^{N}$, for $\phi$ satisfying

$$
\sum_{i=1}^{N} u_{i}^{k}=0 .
$$

Then, there exists a positive constant, $C$, independent of $k$ and $h$ such that $\sup _{k \in \mathbb{N}} \phi\left(u^{k}\right) \leqslant$ $C$. Using Jensen's inequality, for all $k \in \mathbb{N}$, we have that

$$
h \sum_{i=1}^{N} G_{i}\left(u^{k}\right) \leqslant \tilde{C},
$$

where $\tilde{C}$ is positive constant that is independent of $k$ and $h$. This estimate together with (3.1) 3.3 implies that

$$
\sum_{i=1}^{N} \frac{\left|u_{i+1}^{k}-u_{i}^{k}\right|^{2}}{h} \leqslant \bar{C}
$$

for some positive constant $\bar{C}$ that is independent of $k$ and $h$. By a telescoping series argument combined with the Cauchy inequality, for all $l, m \in\{1, \ldots, N\}$, we have

$$
\left|u_{l}^{k}-u_{m}^{k}\right| \leqslant \sum_{i=1}^{N}\left|u_{i-1}^{k}-u_{i}^{k}\right| \leqslant\left(\frac{1}{h}\right)^{\frac{1}{2}}\left(\sum_{i=1}^{N}\left|u_{i-1}^{k}-u_{i}^{k}\right|^{2}\right)^{\frac{1}{2}} \leqslant \bar{C}^{\frac{1}{2}} .
$$

The previous bound combined with (3.8) yields

$$
\max _{1 \leqslant i \leqslant N}\left|u_{i}^{k}\right| \leqslant \bar{C}^{\frac{1}{2}} .
$$


By compactness and by extracting a subsequence if necessary, there exists $u \in \mathbb{R}^{N}$ with $\sum_{i=1}^{N} u_{i}=0$ such that $u^{k} \rightarrow u$ in $\mathbb{R}^{N}$. The continuity of $\phi$ implies that $u$ is a minimizer of $\phi$. Furthermore, (3.7) holds.

Finally, by Lemma 3.3 , we have

$$
A^{N}\left[\begin{array}{c}
\tilde{m} \\
u
\end{array}\right]=\left[\begin{array}{l}
0 \\
0
\end{array}\right]
$$

for $\tilde{m}_{i}=e^{G_{i}(u)}$. By selecting $\bar{H}^{N}$ conveniently and by setting $m_{i}=e^{-\bar{H}^{N}} \tilde{m}_{i}$, we obtain $h \sum_{i=1}^{N} m_{i}=1$. Moreover, the triplet $\left(u, m, \bar{H}^{N}\right)$ satisfies 3.6.

3.5. Monotonicity properties. Next, we prove that the operator $A^{N}$ is monotone.

Lemma 3.6. The operator $A^{N}$ given by 3.5 is monotone in $\mathbb{R}^{N} \times \mathbb{R}^{N}$. More precisely, for all $(m, u),(\theta, v) \in \mathbb{R}_{+}^{N} \times \mathbb{R}^{N}$,

$$
\left\langle A^{N}\left[\begin{array}{c}
m \\
u
\end{array}\right]-A^{N}\left[\begin{array}{l}
\theta \\
v
\end{array}\right],\left[\begin{array}{c}
m \\
u
\end{array}\right]-\left[\begin{array}{l}
\theta \\
v
\end{array}\right]\right\rangle_{\mathbb{R}^{N} \times \mathbb{R}^{N}} \geqslant 0 .
$$

Proof. Fix $(m, u),(\theta, v) \in \mathbb{R}_{+}^{N} \times \mathbb{R}^{N}$. Using the definition of $A^{N}$ and the fact that $\ln (\cdot)$ is increasing, we obtain

$$
\begin{aligned}
&\left\langle A^{N}\left[\begin{array}{c}
m \\
u
\end{array}\right]-A^{N}\left[\begin{array}{l}
\theta \\
v
\end{array}\right],\left[\begin{array}{l}
m \\
u
\end{array}\right]-\left[\begin{array}{l}
\theta \\
v
\end{array}\right]\right\rangle_{\mathbb{R}^{N} \times \mathbb{R}^{N}} \\
&=\sum_{i=1}^{N}\left[\left(G_{i}(v)-G_{i}(u)+\ln m_{i}-\ln \theta_{i}\right)\left(m_{i}-\theta_{i}\right)+\left(\left(\mathcal{L}_{u}^{*} m\right)_{i}-\left(\mathcal{L}_{v}^{*} \theta\right)_{i}\right)\left(u_{i}-v_{i}\right)\right] \\
& \geqslant \sum_{i=1}^{N}\left[\left(G_{i}(v)-G_{i}(u)\right) m_{i}+\left(\mathcal{L}_{u}^{*} m\right)_{i}\left(u_{i}-v_{i}\right)\right] \\
& \quad+\sum_{i=1}^{N}\left[\left(G_{i}(u)-G_{i}(v)\right) \theta_{i}-\left(\mathcal{L}_{v}^{*} \theta\right)_{i}\left(u_{i}-v_{i}\right)\right] .
\end{aligned}
$$

Moreover, by the periodicity convention, we have that

$$
\begin{aligned}
\sum_{i=1}^{N}\left(\mathcal{L}_{u}^{*} m\right)_{i}\left(u_{i}-v_{i}\right)= & \frac{1}{h} \sum_{i=1}^{N}\left[-\frac{\partial F_{i-1}}{\partial p}\left(\psi_{i-1}(u)\right) m_{i-1}\left(u_{i}-v_{i}\right)+\frac{\partial F_{i}}{\partial p}\left(\psi_{i}(u)\right) m_{i}\left(u_{i}-v_{i}\right)\right] \\
& +\frac{1}{h} \sum_{i=1}^{N}\left[\frac{\partial F_{i}}{\partial q}\left(\psi_{i}(u)\right) m_{i}\left(u_{i}-v_{i}\right)-\frac{\partial F_{i+1}}{\partial q}\left(\psi_{i+1}(u)\right) m_{i+1}\left(u_{i}-v_{i}\right)\right] \\
= & \frac{1}{h} \sum_{i=1}^{N}\left[-\frac{\partial F_{i}}{\partial p}\left(\psi_{i}(u)\right) m_{i}\left(u_{i+1}-v_{i+1}\right)+\frac{\partial F_{i}}{\partial p}\left(\psi_{i}(u)\right) m_{i}\left(u_{i}-v_{i}\right)\right] \\
& +\frac{1}{h} \sum_{i=1}^{N}\left[\frac{\partial F_{i}}{\partial q}\left(\psi_{i}(u)\right) m_{i}\left(u_{i}-v_{i}\right)-\frac{\partial F_{i}}{\partial q}\left(\psi_{i}(u)\right) m_{i}\left(u_{i-1}-v_{i-1}\right)\right] \\
= & \sum_{i=1}^{N}\left[\frac{\partial F_{i}}{\partial p}\left(\psi_{i}(u)\right)\left(\psi_{i}^{1}(u)-\psi_{i}^{1}(v)\right)+\frac{\partial F_{i}}{\partial q}\left(\psi_{i}(u)\right)\left(\psi_{i}^{2}(u)-\psi_{i}^{2}(v)\right)\right] m_{i} \\
= & \sum_{i=1}^{N} \nabla F_{i}\left(\psi_{i}(u)\right) \cdot\left(\psi_{i}(u)-\psi_{i}(v)\right) m_{i} .
\end{aligned}
$$

So, the estimate

$$
\begin{aligned}
\sum_{i=1}^{N}[ & \left.\left(G_{i}(v)-G_{i}(u)\right) m_{i}+\left(\mathcal{L}_{u}^{*} m\right)_{i}\left(u_{i}-v_{i}\right)\right] \\
& =\sum_{i=1}^{N}\left[F_{i}\left(\psi_{i}(v)\right)-F_{i}\left(\psi_{i}(u)\right)-\nabla F_{i}\left(\psi_{i}(u)\right) \cdot\left(\psi_{i}(v)-\psi_{i}(u)\right)\right] m_{i} \geqslant 0
\end{aligned}
$$


follows from the convexity of each $F_{i}$ and from the positivity of each $m_{i}$. Similarly,

$$
\sum_{i=1}^{N}\left[\left(G_{i}(u)-G_{i}(v)\right) \theta_{i}-\left(\mathcal{L}_{v}^{*} \theta\right)_{i}\left(u_{i}-v_{i}\right)\right] \geqslant 0,
$$

which concludes the proof.

Remark 3.7. Because the operator $A^{N}$ is monotone, $\left(u^{N}, m^{N}, \bar{H}^{N}\right)$ solves 3.6 if and only if the condition

$$
\left\langle A^{N}\left[\begin{array}{l}
\theta \\
v
\end{array}\right]-\left[\begin{array}{c}
\bar{H}^{N} \iota \\
0
\end{array}\right],\left[\begin{array}{l}
\theta \\
v
\end{array}\right]-\left[\begin{array}{c}
m^{N} \\
u^{N}
\end{array}\right]\right\rangle_{\mathbb{R}^{N} \times \mathbb{R}^{N}} \geqslant 0
$$

holds for every $(v, \theta) \in \mathbb{R}^{N} \times \mathbb{R}_{+}^{N}$.

Definition 3.8. We say that $A^{N}$ is strictly monotone if 3.9 holds with strict inequality whenever $(m, u),(\theta, v) \in \mathbb{R}_{+}^{N} \times \mathbb{R}^{N}$ satisfy $v \neq u$ and $\sum_{i=1}^{N} v=\sum_{i=1}^{N} u$.

3.6. Uniform estimates. Estimates that do not depend on $N$ play a major role in establishing the convergence of solutions of (3.6) to (1.1). Here, we prove elementary energy estimates that are sufficient to show convergence.

Proposition 3.9. Let $\left(u^{N}, m^{N}, \bar{H}^{N}\right)$ solve (3.6). Further assume that $\sum_{i=1}^{N} u_{i}^{N}=0$. Then, there exists a positive constant, $C$, independent of $N$ such that

$$
\frac{1}{N} \sum_{i=1}^{N}\left(u_{i}^{N}\right)^{2} \leqslant C
$$

and

$$
\left|\bar{H}^{N}\right| \leqslant C
$$

Proof. By Lemma 3.3 , we have that $\phi\left(u^{N}\right) \leqslant C$, where $C=\phi(0)$. Then, arguing as in the proof of Proposition 3.5. we obtain the $\ell^{2}$ bound in 3.12 .

The bound for $\bar{H}^{N}$ is proven in two steps. First, we have

$$
\frac{1}{N} \sum_{i=1}^{N} G_{i}\left(u^{N}\right)=\bar{H}^{N}+\frac{1}{N} \sum_{i=1}^{N} \ln \left(m_{i}^{N}\right) \leqslant \bar{H}^{N}
$$

by Jensen's inequality. Because $G_{i}$ is bounded from below, we obtain

$$
\bar{H}^{N} \geqslant-C
$$

for some constant $C \geqslant 0$ independent of $N$. Second, for each $i \in\{1, \ldots, N\}$, we multiply the $i$ th equation in $(3.6)$ by $m_{i}$ and the $(N+i)$ th equation by $-u_{i}$. Adding the resulting expressions and summing over $i$, we get

$$
\frac{1}{N} \sum_{i=1}^{N} m_{i}\left(G_{i}(u)-\left(\mathcal{L}_{u} u\right)_{i}\right)=\bar{H}^{N}+\frac{1}{N} \sum_{i=1}^{N} m_{i} \ln m_{i} \geqslant \bar{H}^{N}
$$

by Jensen's inequality. By the concavity of $G_{i}(u)$, we have

$$
G_{i}(u)-\left(\mathcal{L}_{u} u\right)_{i}=G_{i}(u)+\left(\mathcal{L}_{u}(0-u)\right)_{i} \leqslant G_{i}(0) .
$$

Hence, $\bar{H}^{N} \leqslant C$ for some constant $C>0$ independent of $N$.

Remark 3.10. The proof of the previous proposition gives an $\ell^{\infty}$ bound for $u_{i}$, not just the $\ell^{2}$ bound in 3.12 . However, the technique used in the proof is one-dimensional since it is similar to the proof of the one-dimensional Morrey's theorem. As stated in the proposition, inequality 3.12 is a discrete version of the Poincaré inequality; this inequality holds in any dimension. Finally, for our purposes, 3.12 is sufficient. 
3.7. Convergence. Here, we show the convergence of solutions of $(3.6)$ to weak solutions of 1.1 .

Proposition 3.11. For $N \in \mathbb{N}$, let $\left(u^{N}, m^{N}, \bar{H}^{N}\right) \in \mathbb{R}^{N} \times \mathbb{R}^{N} \times \mathbb{R}$ be a solution of (3.6). Denote by $\bar{u}^{N}$ the step function in $[0,1]$ that takes the value $u_{i}^{N}$ in the interval $\left[\frac{i-1}{N}, \frac{i}{N}\right]$ for $1 \leqslant i \leqslant N$. Similarly, $\bar{m}^{N}$ is the step function associated with $m^{N}$. Then, up to a (not relabeled) subsequence, $\bar{H}^{N} \rightarrow \bar{H}$ in $\mathbb{R}, \bar{u}^{N} \rightarrow \bar{u}$ in $L^{2}([0,1])$, and $\bar{m}^{N} \rightarrow \bar{m}$ in $\mathcal{P}([0,1])$. Moreover, $(\bar{m}, \bar{u}, \bar{H})$ is a weak solution of 1.1 .

Proof. According to Proposition $3.9,\left|\bar{H}^{N}\right|$ and $\left\|\bar{u}^{N}\right\|_{L^{2}([0,1])}$ are uniformly bounded with respect to $N$. Moreover, $\left\|\bar{m}^{N}\right\|_{L^{1}([0,1])}=1$ by construction. Therefore, there exist $\bar{H} \in \mathbb{R}$, $\bar{u} \in L^{2}([0,1])$, and $\bar{m} \in \mathcal{P}([0,1])$ such that $\bar{H}^{N} \rightarrow \bar{H}$ in $\mathbb{R}, \bar{u}^{N} \rightarrow \bar{u}$ in $L^{2}([0,1])$, and $\bar{m}^{N} \rightarrow \bar{m}$ in $\mathcal{P}([0,1])$, up to a (not relabeled) subsequence.

Select $v, \theta \in C^{\infty}(\mathbb{T})$ satisfying $\theta>0$ and $\int_{\mathbb{T}} \theta \mathrm{d} x=1$. Set $v_{i}^{N}=v\left(\frac{i}{N}\right)$ and $\theta_{i}^{N}=\theta\left(\frac{i}{N}\right)$. Then, by Remark 3.7 .

$$
\begin{aligned}
0 & \leqslant\left\langle A^{N}\left[\begin{array}{l}
\theta^{N} \\
v^{N}
\end{array}\right]-\left[\begin{array}{c}
\bar{H}^{N} \\
0
\end{array}\right],\left[\begin{array}{c}
\theta^{N} \\
v^{N}
\end{array}\right]-\left[\begin{array}{l}
m^{N} \\
u^{N}
\end{array}\right]\right\rangle_{\mathbb{R}^{N} \times \mathbb{R}^{N}} \\
& =O\left(\frac{1}{N}\right)+\left\langle A\left[\begin{array}{l}
\theta \\
v
\end{array}\right]-\left[\begin{array}{c}
\bar{H}^{N} \\
0
\end{array}\right],\left[\begin{array}{l}
\theta \\
v
\end{array}\right]-\left[\begin{array}{c}
\bar{m}^{N} \\
\bar{u}^{N}
\end{array}\right]\right\rangle_{\mathcal{D} \times \mathcal{D}^{\prime}} .
\end{aligned}
$$

The proposition follows by letting $N \rightarrow \infty$ in this last expression.

3.8. A discrete gradient flow. To approximate 3.6 , we consider two approaches. Here, we discuss a gradient-flow approximation. Later, we examine a monotonicity-based method.

The discrete-time gradient flow is

$$
\dot{\boldsymbol{u}}=-\left(\mathcal{L}_{\boldsymbol{u}^{*}} \tilde{\boldsymbol{m}}\right)_{i},
$$

where $\tilde{\boldsymbol{m}}_{i}=e^{G_{i}(\boldsymbol{u})}$. Because $\phi$ is convex, $\phi(\boldsymbol{u}(t))$ is decreasing. Moreover, the proof of proposition (3.5) shows that $\phi$ is coercive on the set $\sum_{i=1}^{N} \boldsymbol{u}_{i}=0$. Note that (3.13) satisfies

$$
\frac{\mathrm{d}}{\mathrm{d} t} \sum_{i=1}^{N} \boldsymbol{u}_{i}=0
$$

Consequently, $\boldsymbol{u}(t)$ is bounded and converges to a critical point of $\phi$. Finally, we obtain a solution to (3.6) by normalizing $\tilde{\boldsymbol{m}}_{i}$.

3.9. Dynamic approximation. We can use the monotonicity of $A^{N}$ to build a contracting flow in $L^{2}$ whose fixed points satisfy $(3.6)$. This flow is

$$
\left[\begin{array}{c}
\dot{\boldsymbol{m}} \\
\dot{\boldsymbol{u}}
\end{array}\right]=-A^{N}\left[\begin{array}{c}
\boldsymbol{m} \\
\boldsymbol{u}
\end{array}\right]+\left[\begin{array}{c}
\bar{H}^{N}(t) \iota \\
0
\end{array}\right],
$$

where $\bar{H}^{N}(t)$ is such that the total mass is preserved; that is,

$$
\sum_{i=1}^{N} \dot{\boldsymbol{m}}_{i}=0
$$

Due to the logarithmic nonlinearity, $\boldsymbol{m}(t)>0$ for all $t$. We further observe that

$$
\frac{\mathrm{d}}{\mathrm{d} t} \sum_{i=1}^{N} \boldsymbol{u}_{i}=0
$$

Moreover, if $\left(\bar{m}^{N}, \bar{u}^{N}, \bar{H}^{N}\right)$ is a solution of 3.6 , then the monotonicity of $A^{N}$ implies that

$$
\frac{\mathrm{d}}{\mathrm{d} t}\left(\left\|\boldsymbol{m}-\bar{m}^{N}\right\|^{2}+\left\|\boldsymbol{u}-\bar{u}^{N}\right\|^{2}\right) \leqslant 0 .
$$


Furthermore, if strong monotonicity holds (see Definition 3.8), the preceding inequality is strict if $(\boldsymbol{m}, \boldsymbol{u}) \neq\left(m^{N}, u^{N}\right)$. In this case, $(\boldsymbol{m}(t), \boldsymbol{u}(t))$ is globally bounded and converges to $\left(m^{N}, u^{N}\right)$. Finally, this implies that $\bar{H}(t)$ converges to $\bar{H}^{N}$.

\section{Numerical RESUlts}

Here, we discuss the implementation of our numerical methods, the corresponding results, and some extensions.

In our numerical examples, we construct $F$ as follows. First, we build

$$
F^{Q}(p, q)=\frac{1}{2}(\max \{p, q, 0\})^{2}
$$

and

We set

$$
F^{D}(p, q, x)= \begin{cases}-b(x) p & \text { if } b(x) \leqslant 0 \\ b(x) q & \text { otherwise }\end{cases}
$$

$$
F(p, q, x)=F^{Q}(p, q)+F^{D}(p, q, x)+V(x) .
$$

Then, $F_{i}$ is given by 3.2 .

We implemented our algorithms in MATLAB and Mathematica with no significant differences in performance or numerical results. We present here the computations performed with the Mathematica code. To solve the ordinary differential equations, we used the builtin Mathematica ODE solver with the stiff backward difference formula (BDF) discretization of variable order.

4.1. Gradient flow. For the gradient flow, we took $u(x, 0)=0.2 \cos (2 \pi x)$ as the initial condition for $u$. We used $N=100$. We set $b=0$ and $V(x)=\sin (2 \pi x)$. Figures 11 and 2 feature the evolution of $u$ and $m$, respectively, for $0 \leqslant t \leqslant 1$. We can observe a fast convergence to the stationary solution $u=0$. Figure 3 illustrates the behavior of $m$ at equally spaced times and compares it to the exact solution (in black).

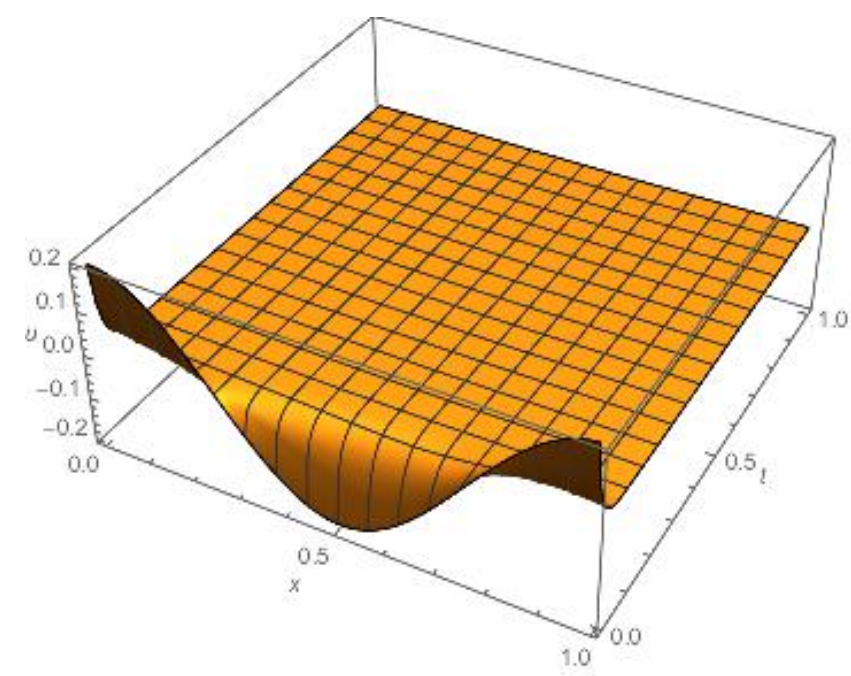

FIG. 1. Gradient flow: $u$

4.2. Monotonic flow. Here, we present the numerical results for the monotonic flow. We set, as before, $b=0$ and $V(x)=\sin (2 \pi x)$. We used $u(x, 0)=0.2 \cos (2 \pi x)$ and $m(x, 0)=$ $1+0.2 \cos (2 \pi x)$ as initial conditions for $u$ and $m$, respectively. As previously, we used $N=100$. Figures 4 and 5 depict the convergence to stationary solutions for $0 \leqslant t \leqslant 10$. Figure 6 compares the values of $m$ at equally spaced times with the stationary solution. Finally, Figures 7 and 8 illustrate the solution $(u, m)$ for the case $b=\cos ^{2}(2 \pi x)$. 


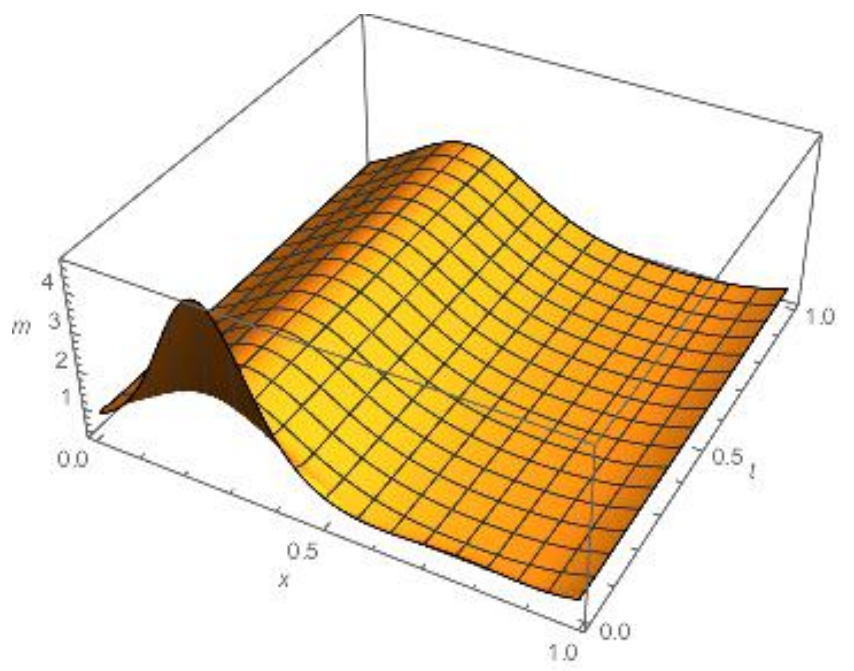

FIG. 2. Gradient flow: $m$

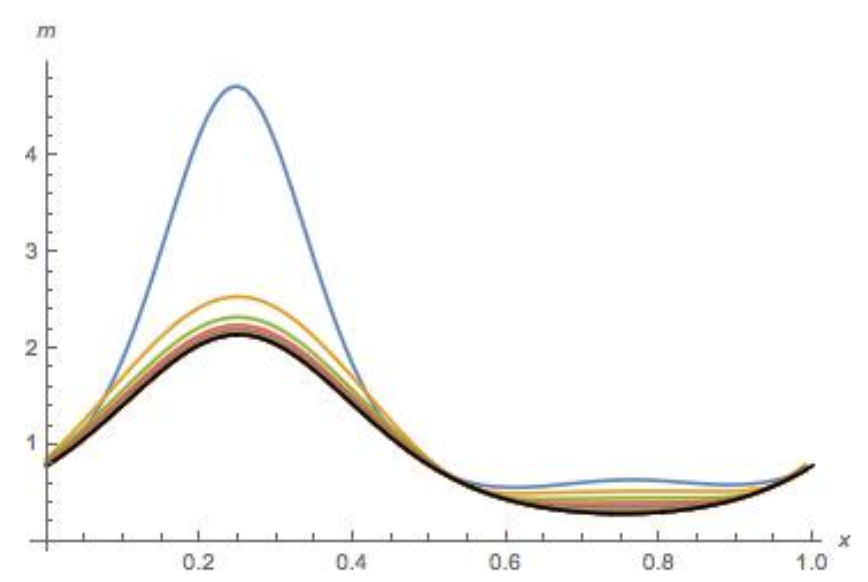

FIG. 3. Gradient flow: $m$ numeric for $0 \leqslant t \leqslant 0.1$ vs. exact (black line)

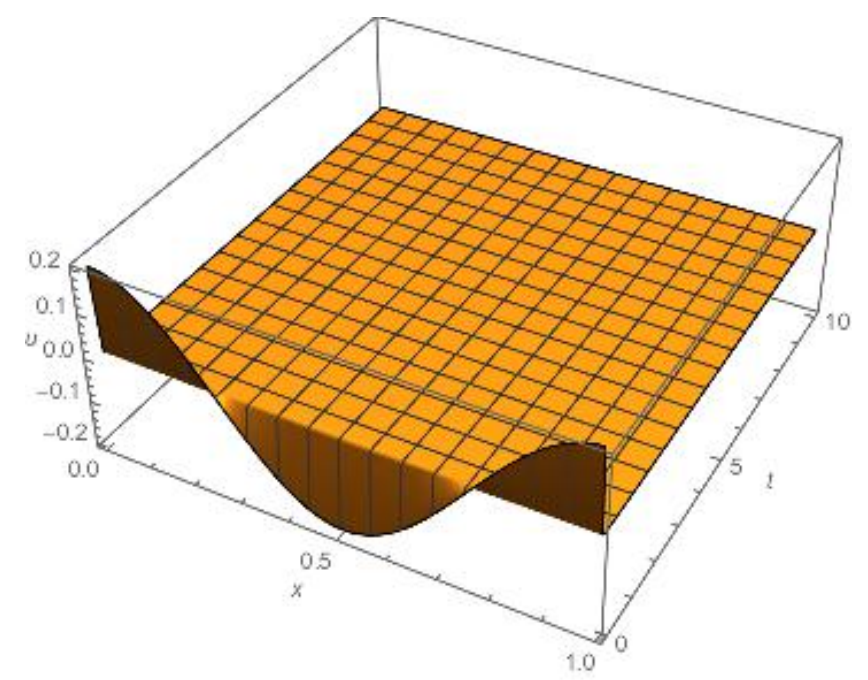

Fig. 4. Monotonic flow: $u$ 


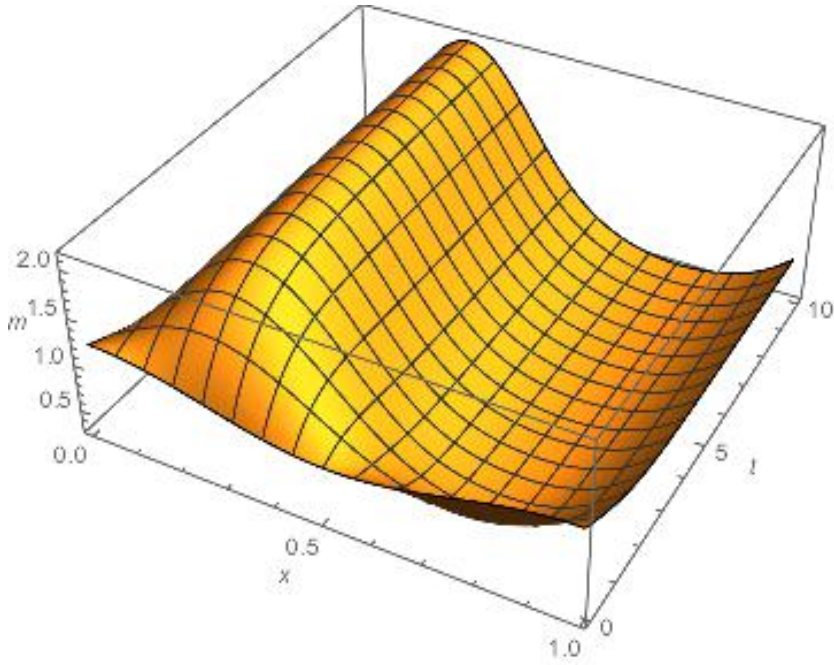

FIG. 5. Monotonic flow: $m$

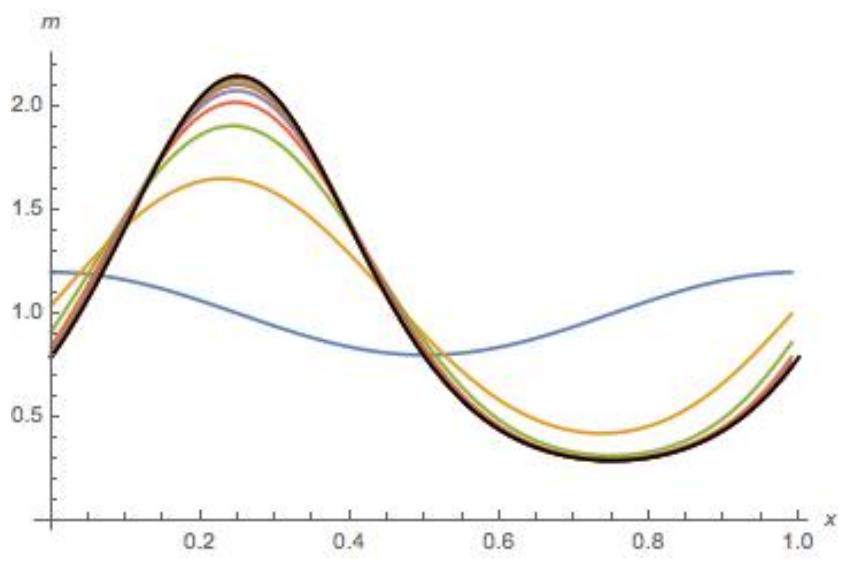

FIG. 6. Monotonic flow: $m$ numerical vs. exact (black line) for $t=0,1, \ldots, 10$

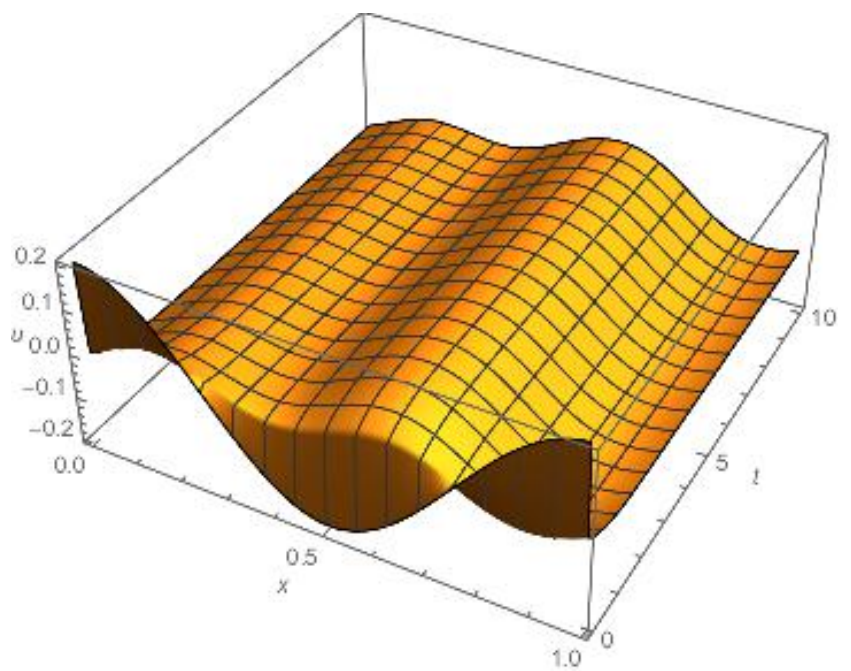

FIG. 7. Monotonic flow: $u$ with $b=\cos ^{2}(2 \pi x)$ 


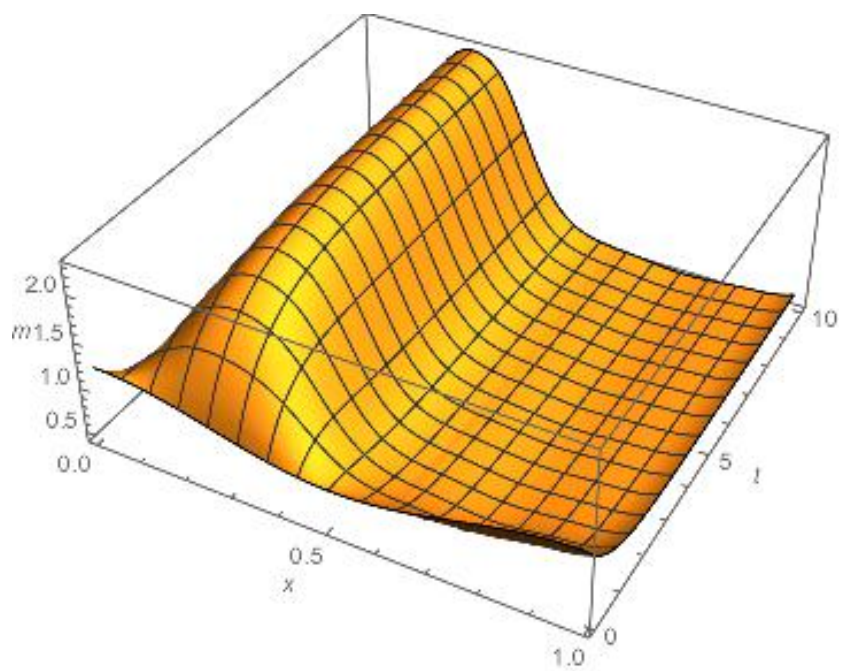

FiG. 8. Monotonic flow: $m$ with $b=\cos ^{2}(2 \pi x)$

4.3. Application to congestion problems. Our methods are not restricted to 1.1 nor to one-dimensional problems. Here, we consider the congestion problem (2.1) and present the corresponding numerical results. We examine higher-dimensional problems in the next section.

The congestion problem (2.1) satisfies the monotonicity condition (see 28). Moreover, this problem admits the same explicit solution as 1.1 with $b=0$. We chose $V(x)=$ $\sin (2 \pi x)$, for comparison.

We took the same initial conditions as in the previous section and set $N=100$. We present the evolution of $u$ and $m$ in Figures 9 and 10, respectively. In Figure 11, we superimpose the exact solution, $m$, on the numerical values of $m$ at equally spaced times.

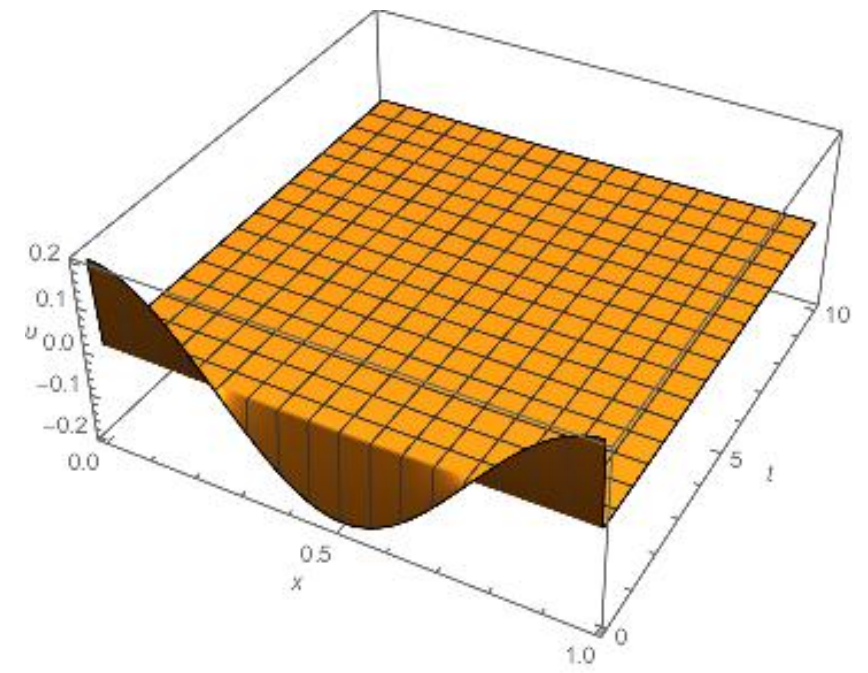

FIG. 9. Congestion model: $u$

4.4. Higher-dimensional examples. The last example, we consider the following twodimensional version of 1.1 :

$$
\left\{\begin{array}{l}
\frac{w_{x}^{2}}{2}+\frac{w_{y}^{2}}{2}+W(x, y)=\ln m+\bar{H} \\
-\left(\theta\left(w_{x}\right)\right)_{x}-\left(\theta\left(w_{y}\right)\right)_{y}=0
\end{array}\right.
$$




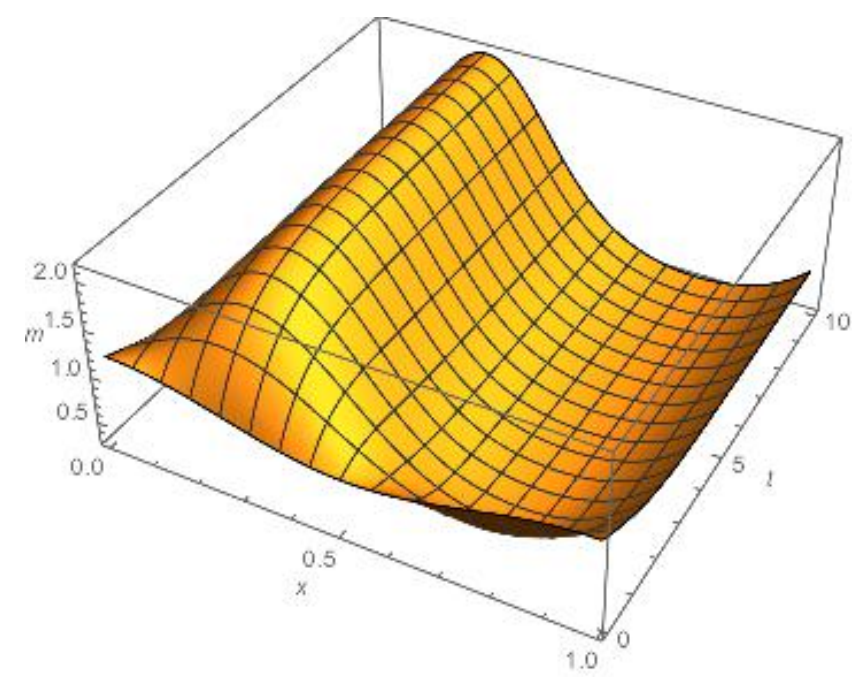

FIG. 10. Congestion model: $m$

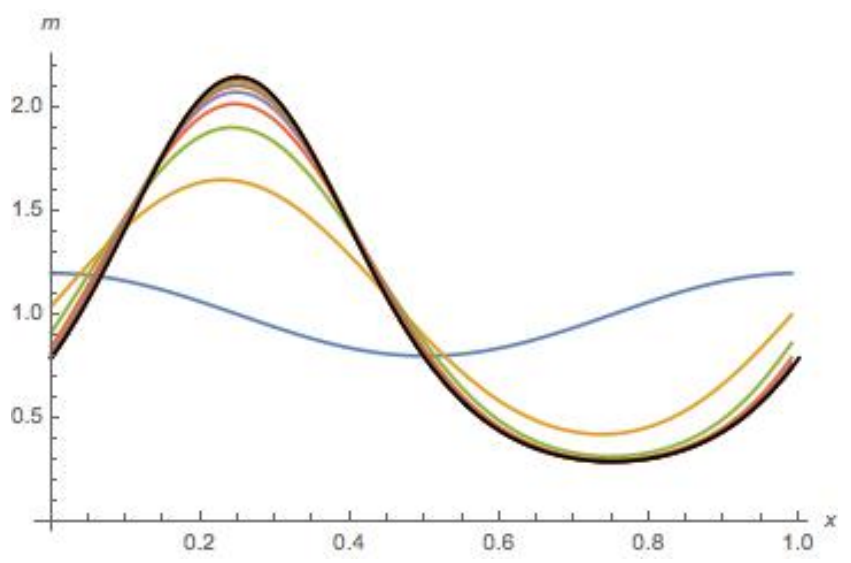

FIG. 11. Congestion model: $m$ numerical vs. exact (black line) for $t=0,1, \ldots, 10$

with $W(x, y)=\sin (2 \pi x)+\sin (2 \pi y)$. Because $W(x, y)=V(x)+V(y)$ for $V(x)=\sin (2 \pi x)$, the solution to (4.1) takes the form $w(x, y)=u(x)+u(y)$ and $\theta(x, y)=m(x)+m(y)$ where $(u, m)$ solves $(1.1)$ with $b=0$.

We chose $w(x, y, 0)=0.4 \cos (x+2 y), \theta(x, y, 0)=1+0.3 \cos (x-3 y)$, and $N=20$. Figure 12 illustrates $\theta$ at $T=50$. The numerical errors for $\theta$ and $w$ are shown in Figures 13 and 14 respectively.

\section{Final Remarks}

Here, we developed two numerical methods to approximate solutions of stationary meanfield games. We addressed the convergence of a discrete version of (1.1), and the convergence to weak solutions through a monotonicity argument. Our techniques generalize to discretized systems that are monotonic, and that admit uniform bounds with respect to the discretization parameter.

In the cases we considered, our methods approximate well the exact solutions. While the gradient flow is considerably faster than the monotonic flow, this last method applies to a wider class of problems.

We selected a simple model for illustration purposes. In our numerical examples, however, we illustrated the convergence of the schemes in higher-dimensional problems and congestion MFG problems. Furthermore, our results can be easily extended to related problems 


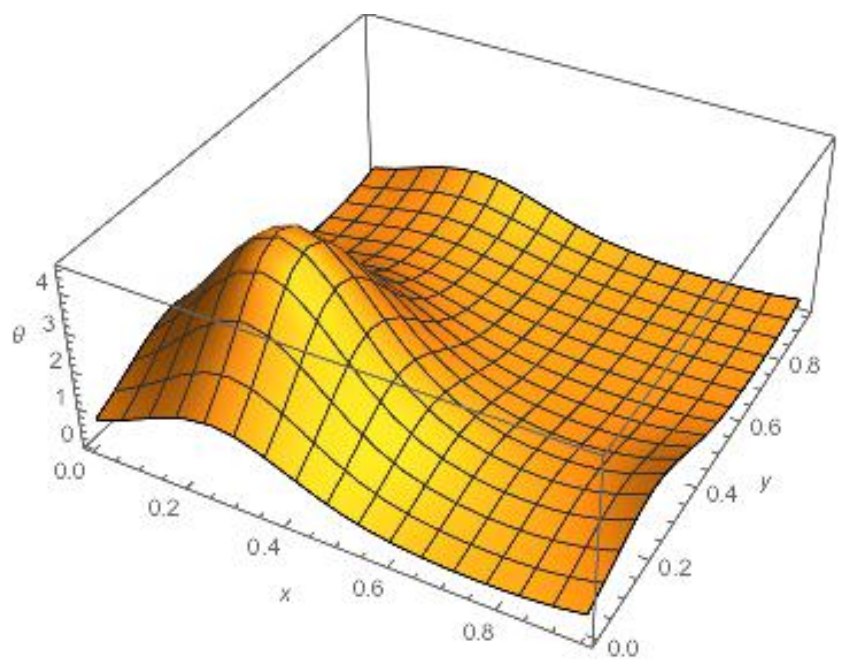

FIG. 12. Two-dimensional problem: numeric $\theta$ at $T=50$

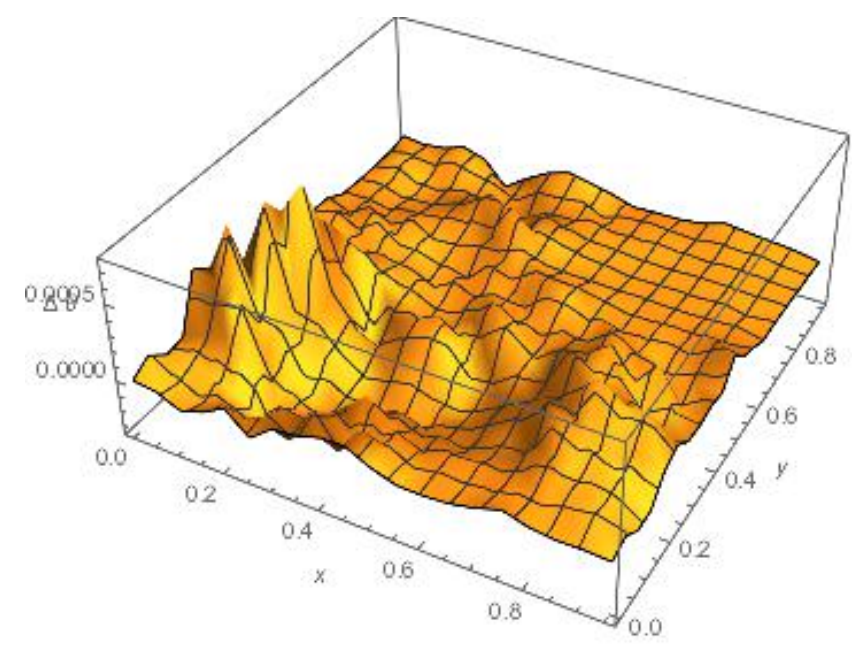

FIG. 13. Two-dimensional problem: $\theta$ numerical error

- higher-dimensional cases, second-order MFG, or non-local (monotonic) problems. Additionally, our methods provide a natural guide for two future research directions. The first is the development of a general theory of convergence for monotone schemes and the extension of our methods to mildly non-monotonic MFG. The second is the study of time-dependent MFG. This last direction is particularly relevant since the coupled structure of MFG and the initial-terminal conditions that are usually imposed make these problems very challenging from the numerical point of view.

\section{REFERENCES}

[1] Y. Achdou. Finite difference methods for mean field games. In Hamilton-Jacobi Equations: Approximations, Numerical Analysis and Applications, pages 1-47. Springer, 2013.

[2] Y. Achdou, F. Camilli, and I. Capuzzo-Dolcetta. Mean field games: numerical methods for the planning problem. SIAM J. Control Optim., 50(1):77-109, 2012.

[3] Y. Achdou and I. Capuzzo-Dolcetta. Mean field games: numerical methods. SIAM J. Numer. Anal., 48(3):1136-1162, 2010.

[4] Y. Achdou and V. Perez. Iterative strategies for solving linearized discrete mean field games systems. Netw. Heterog. Media, 7(2):197-217, 2012.

[5] Y. Achdou and A. Porretta. Convergence of a finite difference scheme to weak solutions of the system of partial differential equation arising in mean field games. Preprint, 2015. 


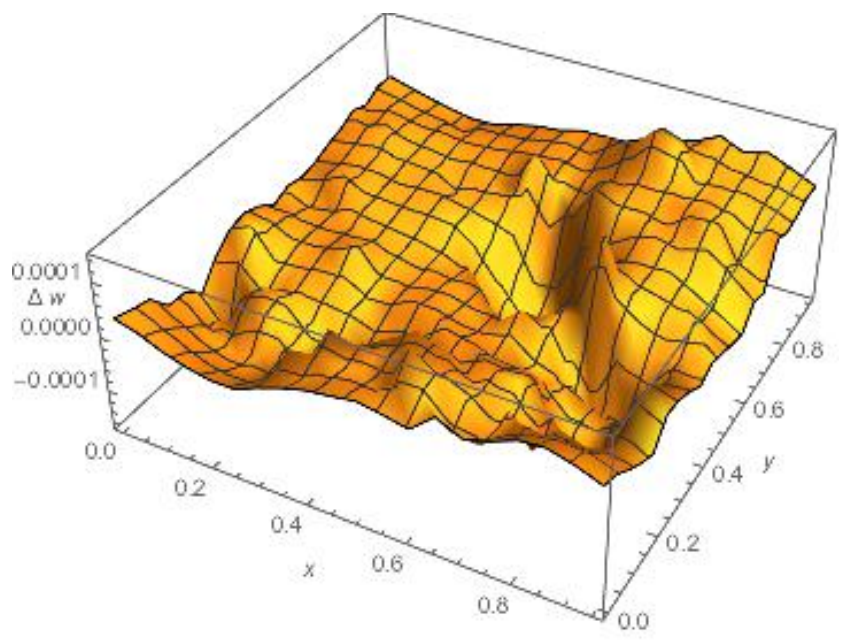

Fig. 14. Two-dimensional problem: $w$ numerical error

[6] G. Barles and P. E. Souganidis. Convergence of approximation schemes for fully nonlinear second order equations. Asymptotic Anal., 4(3):271-283, 1991.

[7] A. Bensoussan, J. Frehse, and P. Yam. Mean field games and mean field type control theory. Springer Briefs in Mathematics. Springer, New York, 2013.

[8] F. Camilli and F. Silva. A semi-discrete approximation for a first order mean field game problem. Netw. Heterog. Media, 7(2):263-277, 2012.

[9] Fabio Camilli, Adriano Festa, and Dirk Schieborn. An approximation scheme for a Hamilton-Jacobi equation defined on a network. Appl. Numer. Math., 73:33-47, 2013.

[10] P. Cardaliaguet. Notes on mean-field games. 2011.

[11] P. Cardaliaguet and P. J. Graber. Mean field games systems of first order. ESAIM Control Optim. Calc. Var., 21(3):690-722, 2015.

[12] P. Cardaliaguet, P. J. Graber, A. Porretta, and D. Tonon. Second order mean field games with degenerate diffusion and local coupling. NoDEA Nonlinear Differential Equations Appl., 22(5):1287-1317, 2015.

[13] E. Carlini and F. J. Silva. A fully discrete semi-Lagrangian scheme for a first order mean field game problem. SIAM J. Numer. Anal., 52(1):45-67, 2014.

[14] L. C. Evans. Partial Differential Equations. Graduate Studies in Mathematics. American Mathematical Society, 1998.

[15] L. C. Evans. Some new PDE methods for weak KAM theory. Calc. Var. Partial Differential Equations, 17(2):159-177, 2003.

[16] L. C. Evans. Further PDE methods for weak KAM theory. Calc. Var. Partial Differential Equations, 35(4):435-462, 2009.

[17] R. Ferreira and D. Gomes. Existence of weak solutions for stationary mean-field games through weak solutions. Preprint.

[18] D. Gomes, R. Iturriaga, H. Sánchez-Morgado, and Y. Yu. Mather measures selected by an approximation scheme. Proc. Amer. Math. Soc., 138(10):3591-3601, 2010.

[19] D. Gomes, S. Patrizi, and V. Voskanyan. On the existence of classical solutions for stationary extended mean field games. Nonlinear Anal., 99:49-79, 2014.

[20] D. Gomes and E. Pimentel. Local regularity for mean-field games in the whole space. To appear in Minimax Theory and its Applications, 2015.

[21] D. Gomes, E. Pimentel, and H. Sánchez-Morgado. Time dependent mean-field games in the superquadratic case. To appear in ESAIM: Control, Optimisation and Calculus of Variations.

[22] D. Gomes, E. Pimentel, and H. Sánchez-Morgado. Time-dependent mean-field games in the subquadratic case. Comm. Partial Differential Equations, 40(1):40-76, 2015.

[23] D. Gomes, G. E. Pires, and H. Sánchez-Morgado. A-priori estimates for stationary mean-field games. Netw. Heterog. Media, 7(2):303-314, 2012.

[24] D. Gomes and H. Sánchez Morgado. A stochastic Evans-Aronsson problem. Trans. Amer. Math. Soc., 366(2):903-929, 2014.

[25] D. Gomes, R. M. Velho, and M.-T. Wolfram. Dual two-state mean-field games. Proceedings CDC 2014, 2014.

[26] D. Gomes, R. M. Velho, and M.-T. Wolfram. Socio-economic applications of finite state mean field games. Philos. Trans. R. Soc. Lond. Ser. A Math. Phys. Eng. Sci., 372(2028):20130405, 18, 2014.

[27] D. Gomes and V. Voskanyan. Short-time existence of solutions for mean-field games with congestion. Preprint. 
[28] D. A. Gomes and H. Mitake. Existence for stationary mean-field games with congestion and quadratic Hamiltonians. NoDEA Nonlinear Differential Equations Appl., 22(6):1897-1910, 2015.

[29] D. A. Gomes and S. Patrizi. Obstacle mean-field game problem. Interfaces Free Bound., 17(1):55-68, 2015.

[30] D. A. Gomes and E. Pimentel. Time-Dependent Mean-Field Games with Logarithmic Nonlinearities. SIAM J. Math. Anal., 47(5):3798-3812, 2015.

[31] D. A. Gomes and J. Saúde. Mean field games models-a brief survey. Dyn. Games Appl., 4(2):110-154, 2014.

[32] O. Guéant. Mean field games equations with quadratic Hamiltonian: a specific approach. Math. Models Methods Appl. Sci., 22(9):1250022, 37, 2012.

[33] O. Guéant. Mean field games with a quadratic Hamiltonian: a constructive scheme. In Advances in dynamic games, volume 12 of Ann. Internat. Soc. Dynam. Games, pages 229-241. Birkhäuser/Springer, New York, 2012.

[34] O. Guéant. New numerical methods for mean field games with quadratic costs. Netw. Heterog. Media, $7(2): 315-336,2012$.

[35] M. Huang, P. E. Caines, and R. P. Malhamé. Large-population cost-coupled LQG problems with nonuniform agents: individual-mass behavior and decentralized $\epsilon$-Nash equilibria. IEEE Trans. Automat. Control, 52(9):1560-1571, 2007.

[36] M. Huang, R. P. Malhamé, and P. E. Caines. Large population stochastic dynamic games: closed-loop McKean-Vlasov systems and the Nash certainty equivalence principle. Commun. Inf. Syst., 6(3):221$251,2006$.

[37] J.-M. Lasry and P.-L. Lions. Jeux à champ moyen. I. Le cas stationnaire. C. R. Math. Acad. Sci. Paris, 343(9):619-625, 2006

[38] J.-M. Lasry and P.-L. Lions. Jeux à champ moyen. II. Horizon fini et contrôle optimal. C. R. Math. Acad. Sci. Paris, 343(10):679-684, 2006.

[39] J.-M. Lasry and P.-L. Lions. Mean field games. Jpn. J. Math., 2(1):229-260, 2007.

[40] A. R. Mészáros and F. J. Silva. A variational approach to second order mean field games with density constraints: The stationary case. J. Math. Pures Appl. (9), 104(6):1135-1159, 2015.

[41] A. M. Oberman. Convergent difference schemes for degenerate elliptic and parabolic equations: Hamilton-Jacobi equations and free boundary problems. SIAM J. Numer. Anal., 44(2):879-895 (electronic), 2006.

[42] E. Pimentel and V. Voskanyan. Regularity for second-order stationaty mean-field games. To appear in Indiana University Mathematics Journal.

[43] A. Porretta. On the planning problem for the mean field games system. Dyn. Games Appl., 4(2):231256, 2014.

[44] A. Porretta. Weak solutions to Fokker-Planck equations and mean field games. Arch. Ration. Mech. Anal., 216(1):1-62, 2015.

[45] F. Santambrogio. A modest proposal for MFG with density constraints. Netw. Heterog. Media, 7(2):337347, 2012.

[46] V. K. Voskanyan. Some estimates for stationary extended mean field games. Dokl. Nats. Akad. Nauk Armen., 113(1):30-36, 2013.

N. Almulla, University of Dammam, College of Science, King Faisal Road, Dammam Saudi ARABia

R. Ferreira and D. Gomes, King Abdullah University of Science and Technology (KAUST), CEMSE Division \& KAUST SRI, Center for Uncertainty Quantification in Computational Science And Engineering, Thuwal 23955-6900, SAudi Arabia.

E-mail address, N. Almulla: nalmulla@uod.edu.sa

E-mail address, R. Ferreira: rita.ferreira@kaust.edu.sa

E-mail address, D. Gomes: diogo.gomes@kaust.edu.sa 